\title{
Jänschwalde und die Gliederung des Saale-Komplexes - ein Kommentar zum Beitrag von WERNER Nowel
}

\section{Lothar Lippstreu \& Werner STACKebrandT*)}

Lippstreu, L. \& STACKebrandT, W. (2003): Jänschwalde und die Gliederung des Saale-Komplexes - ein Kommentar zum Beitrag von Werner Nowel. - Eiszeitalter und Gegenwart, 52: 84-90; Hannover.

Keywords: Quaternary, stratigraphy, Saale glaciation, North Germany, Lausitz

Kurzfassung: Die Ansichten der Autoren zur Stratifizierung des Saale-Komplexes stehen teilweise im Widerspruch zu den Auffassungen von Werner Nowel. Besondere Bedeutung kommt hierfür dem Tranitz-Fluviatil als litho- und klimastratigraphischer Leithorizont $\mathrm{zu}$, der von uns unterhalb des saalezeitlichen Drentheglazials eingestuft wird im Gegensatz zur Nowelschen Einstufung oberhalb des saalezeitlichen Drentheglazials.

\section{[Jänschwalde and the subdivision of the Saale Complex - a comment to the article of WERNER NOWEL]}

Abstract: The author's view of the stratification of the glacial Saale Complex partly contrasts to that given by Werner Nowel. As a lithological and climatic marker horizon the Tranitz-Fluviatil is positioned beneath the Saalean Drenthe glacial instead of superimposing the Saalean Drenthe glacial sensu Nowel.

Die Jänschwalder Tagebaulandschaft in der Niederlausitz mit ihren aktiven und aufgelassenen, aber derzeit noch zugänglichen Baggerböschungen war anlässlich der Potsdamer DEUQUA-Tagung im August 2002 erneut Ziel einer geologischen Fachexkursion (vgl. STACKEBRANDT et al. 2002). Auf Bitte der Redaktion von Eiszeitalter und Gegenwart und insbesondere auf ausdrücklichen Wunsch des damaligen Vorsitzenden der DEUQUA, Herrn Prof. Dr. W. Schirmer - Teilnehmer der Exkursion A2 -, werden im Folgenden die während dieser

*) Anschriften der Verfasser: Dipl.-Geol. L. Lippstreu, Dr. W. STACKeBRANDT, Landesamt für Geowissenschaften und Rohstoffe Brandenburg, Stahnsdorfer Damm 77, D-14532 Kleinmachnow. E-Mail: stackebrandt@lgrb.de
Exkursion präsentierten Aufschlüsse und sich daraus ergebende Konsequenzen für die stratigraphische Gliederung insbesondere der saalezeitlichen Schichtenfolge kommentiert. Sie stehen zumindest teilweise im Widerspruch zu den im voranstehenden Beitrag von W. Nowel gezogenen Schlussfolgerungen zur stratigraphischen Bewertung und Zuordnung der u. a. geschiebeanalytisch untersuchten Grundmoränen dieses Gebietes.

Mit dem sukzessiven Fortschreiten des Tagebaus Jänschwalde nach Norden - zur Zeit im Bereich der Hornoer Hochfläche bauend - entstanden während der letzten 25 Jahre beeindruckende Aufschlüsse im quartären Deckgebirge, die trotz ihrer unterschiedlichen Interpretation eine überregionale $\mathrm{Be}$ deutung für die Gliederung des Mittelpleistozäns in Ostbrandenburg erlangt haben. Während der Exkursion A2 konnten entlang dieser Süd-NordTraverse noch einmal einige aussagekräftige Quartärprofile aus dem Klinger Becken (Südrandschlauch mit dem Eem von Klinge) und der Weissagker Hochfläche (Ostrandschlauch westlich Mulknitz) sowie nördlich des Baruther Urstromtales von der aktiven Baggerböschung an der Hornoer Hochfläche gezeigt werden. Sie gestatten die folgende generelle Aussage:

Sowohl auf der Hornoer Hochfläche wie auch südlich des Baruther Urstromtals stehen oberflächig in \pm gleichem Höhenniveau ( $+80-90 \mathrm{~m}$ ü. $\mathrm{NN}$ ) saalezeitliche Grundmoränen an. Auf der Weissagker und Dubrauer Hochfläche werden sie von fluviatilen Sanden bis Kiesen mit südlichem Geröllbestand unterlagert, die als so genanntes „Tranitzer Fluviatil" (qsTR) in die Literatur eingingen (HeLLWIG 1975) und einem fossilen Neißelauf zugeordnet werden. Dieses Tranitz-Fluviatil hat für die Stratifizierung der quartären Schichtenfolgen des gesamten Forst-Cottbuser Raumes (Tagebaugebiet Jänschwalde) eine wichtige lithostratigraphische Funktion erlangt. HeLlWIG (1975) selbst ordnete es 
auf der Basis seiner geschiebeanalytischen Untersuchungsergebnisse, gewonnen zunächst überwiegend aus Bohrungen, in den Zeitraum zwischen SII- und SIII-Glazial sensu CEPEK mit einem Hinweis auf die stratigraphische Nähe zur CEPEKschen Rügen-Warmzeit (HellWIG 1975: 1085-88) ein. Entsprechend den in den letzten Jahren von W. Nowel für die Niederlausitz erarbeiteten Stratigraphieanpassungen und -korrelationen, die sich sowohl mit Teilen der BEHRENDTschen Überlegungen dazu (BEHRENDT 1998) in Übereinstimmung bringen lassen, als sich auch, was das glaziär entwickelte Obere Saale anbelangt, unseren Vorstellungen angenähert haben, wird das tranitzfluviatile Sediment jetzt von Nowel $(1996,2003)$ in seiner Gesamtheit, also untere und obere Folge sensu Hellwig, zwischen Drenthe und Warthe eingeordnet, $d$. h. auch weiterhin als ein intrasaalezeitliches Fluviatil (innerhalb des Oberen Saale) interpretiert.

Demgegenüber konnte im Ergebnis unserer detaillierten Kartier- und Untersuchungsarbeiten nachgewiesen werden, dass das Tranitz-Fluviatil an der Ostböschung des so genannten Südrandschlauches (südlicher Randbereich der Weissagker Hochfläche bei Gosda) von der Grundmoräne des 1. saalezeitlichen Inlandeisvorstoßes begraben bzw. abgeschnitten wird. Es muss also auf Grund von relativzeitlichen Kriterien älter als Drenthe (qsD) sein (Argumente siehe u. a. bei Lippstreu et al. 1995, 1997 und Lippstreu 1998, 2002).

Die von uns vorgenommene Zuordnung des hangenden Geschiebemergels erfolgte geschiebeanalytisch nach der Methode CEPEK und nach TGL 25232 (1980) sowie durch überlagernde jüngere Schmelzwassersande und Beckenentwicklungen mit auflagernden Eem-Vorkommen. Das Liegende des Tranitz-Fluviatils bildet im Südrandschlauch eine nur wenige Meter mächtige, verwitterte und entkalkte sowie an ihrer Oberfläche olivgrün bis grünlichblau gefärbte Grundmoräne mit zergrusenden nordischen Kristallingeschieben. Sie wird bei LippSTREU et al. (1994 und später) in die Elster-Kaltzeit gestellt, ihre Verwitterung als das Ergebnis einer Warmzeit aus dem Zeitabschnitt Holstein-Frühsaale-Komplex gewertet. Für das Tranitz-Fluviatil ergibt sich somit die stratigraphische Position postholstein- bis frühdrenthezeitlich und wurde daher von uns in das so genannte Mittlere Fluviatil sensu EISSMANN (Unteres Saale) gestellt, und zwar das untere, überwiegend feinerkörnige Tranitz („Klinger Fluviatil") in die Fuhne-Kaltzeit, hingegen das obere, gröberkörnige Tranitz, der eigentliche "Neißeschotter", in den post-dömnitzzeitlichen Abschnitt.

Gestützt werden die von LIPPSTreu und seinen Kollegen vorgenommenen Zuordnungen der Schichtenfolgen im Südrandschlauch durch OSLDatierungen an Proben aus verschiedenen Niveaus der fluviatilen Sedimentkörper (KRBETSCHEK \& STOLz 1994) sowie durch eine Serie von quantitativen Tonmineral-Untersuchungen an den seinerzeit entlang des Südrandschlauches noch gut zugänglichen Grundmoränen (LUCKERT\& THIEKE 2000).

Was die bei etwa +70 bis $+80 \mathrm{~m}$ NN liegende Oberkante des Tranitz-Fluviatils betrifft, so sei darauf verwiesen, dass weiter südlich gelegene, zeitlich adäquat einzuordnende fluviatile Bildungen durchaus vergleichbare Oberkantenwerte zeigen. So liegt z. B. das Holstein von Lug (TK 4349), das bislang einzige pollenanalytisch gesicherte Holstein-Profil des Niederlausitzer Altmoränengebietes (SEIFERT 1989), mit seiner Oberkante bei $+74 \mathrm{~m}$ ü. NN und die OK der darüber entwickelten frühsaalezeitlichen fluviatilen kiesigen Sande bei +97 m ü. NN (UNGER et al. 1995), höhenmäßig im Übrigen hervorragend passend zum ebenfalls frühsaalezeitlichen „Pritzener Fluviatil“ (LipPSTREu et al. 1995). Und vom Jänschwalder Gebiet ca. 60 km weiter nach NNE liegt die von der dortigen Drenthemoräne gekappte Oberfläche eines bei Müllrose (3752 der TK 25) über pollenanalytisch belegtem Dömnitz (KöHLER \& ERD 1980, unveröff.) erbohrten Fluviatils immerhin noch bei rund $+25 \mathrm{~m}$ NN. Damit soll zwar nichts über die Flusszugehörigkeit ausgesagt werden - das Material war für Geröllanalysen zu feinkörnig -, doch auch Neiße und Oder müssen irgendwie ihren Weg nach Norden bzw. Nordwesten genommen haben, woraus sich dann ein theoretischer Gefällewert von $0,9 \mathrm{~m} / \mathrm{km}$ ergeben würde.

Im Gegensatz zu den stratigraphischen Zuordnungen bei Lippstreu et al. (1995, 1994) wird von 
Cepek, Hellwig \& Nowel (zuletzt 1994) und auch von Nowel in mehreren Publikationen die weitflächig um Gosda-Weissagk-Dubrau zutage gehende Grundmoräne als Saale-III sensu CEPEK interpretiert und die im Liegenden des Fluviatils lagernde Grundmoräne ins Saale-I-Glazial gestellt, was bei ihnen folglich zwangsläufig zu der o. g. stratigraphischen Position für das Tranitz-Fluviatil führt.

In lithologisch sehr ähnlichen Entwicklungen lässt sich die komplex untersuchte Schichtenfolge des Südrandschlauches nach Norden über die 1989 von LipPSTREU und seinen Kollegen kartierten Ostrandprofile bei Gosda - verwitterte saalezeitliche Grundmoräne über $20 \mathrm{~m}$ mächtigem Tranitz-Fluviatil über durchgehend entkalkter elsterzeitlicher Grundmoräne über Miozän - hin zum Ostrandschlauch bei Mulknitz auf der Weissagker Hochfläche verfolgen. Und letztlich sind auch auf der nördlich des Baruther Urstromtales gelegenen Hornoer Hochfläche vergleichbar ausgebildete Schichten durch den Tagebauvortrieb angeschnitten, die sich nach KüHNER (Exkursionsführung DEUQUA, A2, s.o.) problemlos mit den weiter südlicher gelegenen Profilen korrelieren lassen.

Die Aufschlüsse an der Tagebauböschung westlich Mulknitz (4253 der TK 25) gewähr(t)en einen Einblick in den Flankenbereich der in der ElsterKaltzeit subglaziär-hydromechanisch angelegten, tief ins Miozän eingeschnittenen Dubrau-Bohrauer Rinne und in den Aufbau und die Lagerungsverhältnisse ihrer Sedimentfüllung. Die im Wesentlichen nur auf die Vorschnittböschung beschränkte saalezeitliche Schichtenfolge scheint sich hier auf das Tranitz-Fluviatil und eine überlagernde Grundmoräne zu reduzieren, so dass sich auch hier die für das Jänschwalder Gebiet vielmals „beschworene“ Superposition der drei saalezeitlichen Glazialfolgen Saale-I, Saale-II und Saale-III sensu CEPEK bzw. nach neuem Verständnis Drenthe und Warthe nur schwerlich wird beweisen lassen. Leider wird der Ostrandschlauch gegenwärtig über eine Bandstraße mit Abraummaterial von der Hornoer Hochfläche verkippt, so dass die Teilnehmer der DEUQUAExkursion A2 wohl die Letzten gewesen sein dürften, die die bereits mehrfach auf Exkursionen vorgestellten und nochmals von der LAUBAG mit schwerem Gerät vorbereiteten Profile haben in Augenschein nehmen können.

Unterhalb der Hauptarbeitsebene (HAE) war noch im Sommer 2002 der annähernd Nord-Süd streichende Flankenbereich der Dubrau-Bohrauer Rinne aufgeschlossen. Die abschnittsweise in die HAE einbiegende, stark eisenverkrustete Kontaktfläche der Rinne zum westlich angrenzenden Miozän (Brieske-Formation) konnte noch etwa $200 \mathrm{~m}$ weit nach Süden verfolgt werden. An mehreren Stellen gestatte(te)n großflächige, von der HAE ausgehende Böschungsabrisse und Wasserrisse einen Blick auf die Sedimentfüllung der Rinne. Diese setzt sich hier am unmittelbaren Rinnenrand im wesentlichen aus staffelartig in die Rinne hereingebrochenen Tertiärsedimenten zusammen. Das angrenzende Miozän zeigt sich hingegen nahezu ungestört. Zum Hangenden hin und zugleich auch rinneneinwärts folgt eine nahezu chaotisch gelagerte Abfolge aus verlagertem und verstürztem Tertiär, quartären Sanden und Beckenschluffen sowie zerbrochenen und anscheinend übereinander gestapelten Moränenpaketen. Darüber lagert im wesentlichen Geschiebemergel. Er ist flintreich, sehr fest und hangaufwärts in mehreren kleinen Abrissen und Aufschürfungen aufgeschlossen. Die oberen Profilabschnitte des Geschiebemergels sowie die gesamte hangende Abfolge bis zur Geländeoberkante waren 1999 durch Schurfarbeiten sogar über eine große Fläche freigelegt und so der direkten Beobachtung zugänglich. Nach den Darstellungen bei CEPEK et al. (1994, Abb. 3, Schnitt 3) sowie Nowel (2003: Abb. 2) nimmt dieser im Rinnenzentrum mehrere Dekameter mächtige, von Sandmitteln durchsetzte Geschiebemergel den gesamten mittleren und oberen Profilabschnitt der DubrauBohrauer Rinne ein. Er wird überlagert von einem flow till und feinsandig-schluffigen glazilimnischen Bildungen; über diesen wiederum folgt eine an ihrer Oberfläche entkalkte Grundmoräne mit olivgrün bis grünlichblau gefärbtem, altangelegtem $\mathrm{Gr}$ Horizont. Flow till und Beckenbildung sind jeweils nur wenige Meter mächtig, die darüber folgende Grundmoräne erreicht nach Nowel eine maximale Mächtigkeit von $6 \mathrm{~m}$.

Die Lagerungsverhältnisse der gesamten Abfolge werden in den Nowelschen Ausführungen insge- 
samt als relativ ungestört beschrieben. Nach unseren Beobachtungen und Aufzeichnungen hingegen ist die gesamte unterhalb des Tranitz-Fluviatils aufgeschlossene Abfolge stark glazigen und allem Anschein nach auch gravitativ deformiert. Eine "nahe$\mathrm{zu}$ ungestörte Lagerung" war von uns in den freigelegten Profilabschnitten und Böschungsabrissen zu keiner Zeit zu erkennen. Vielmehr zeigt die gesamte Geschiebemergel-Schluff (flow till)Geschiebemergel/-lehm-Abfolge ein mehr oder weniger steiles Einfallen nach Nordwesten und wird etwa im Planum der oberen Arbeitsebene „brettartig" sowie mit deutlicher Diskordanz vom glazigen ungestörten Tranitz-Fluviatil überlagert. Dabei wird die Deformation nicht nur in den feingeschichteten Ablagerungen deutlich, sondern gibt sich auch in der paketweise markant brekziösen Struktur des sehr mächtigen, „unteren“ Geschiebemergels zu erkennen.

Die für das Mulknitzer Profil von Nowel vorgenommene stratigraphische Zuordnung der im Liegenden des Tranitz-Fluviatils aufgeschlossenen Quartärsequenz („Obere Rinnenfolge“) erfolgte auf der Basis der 1994 von Hellwig durchgeführten Kleingeschiebeanalysen. Hiernach werden die „obere“, oberflächig entkalkte Grundmoräne als saalezeitliche Grundmoräne vom Typ SII sensu CEPEK und der "untere", mächtige Geschiebemergel als saalezeitliche Grundmoräne vom Typ SI sensu Cерек (jetzt beide mit Drenthe parallelisiert) ausgewiesen. Die zwischen beiden Geschiebemergeln lagernden Bildungen (flow till und Beckensedimente) werden von Nowel in die SII-Vorschüttphase eingeordnet. Inwieweit die zwei 1997 von NOWEL „im ausgespülten Saale-I-Till“ aufgesammelten Exemplare von Viviparus diluvianus KuntH als „Absicherung“ der saalezeitlichen Position der unter dem Tranitz-Fluviatil aufgeschlossenen Abfolge gelten können, ist ob ihrer fraglichen Herkunft zunächst noch in Zweifel zu ziehen.

Auch die Zuordnung der „Hangendfolge“, also des Tranitz-Fluviatils und der auf der Weißagker Hochfläche zutage gehenden Grundmoräne, gründet sich bei Nowel auf entsprechende Probenuntersuchungen von HeLlWIG. Mit Geröllanalysen wird die Zugehörigkeit des Tranitz-Fluviatils zum Flusssystem der Lausitzer Neiße bestätigt und
Kleingeschiebeanalysen aus den nicht verlehmten Profilabschnitten der "Oberflächenmoräne" erbrachten Spektren, die für Nowel $(1996,2003)$ und auch schon für CEPEK et al. (1994) eine Zuordnung zur saalezeitlichen Grundmoräne vom Typ SIII sensu Cepé (jetzt parallelisiert mit qWA) rechtfertigen.

Nach unseren Untersuchungen, u. a. auch aufgrund von Kleingeschiebeanalysen, gibt es keinen Grund, die „Oberflächenmoräne“ des Mulknitzer Ostrandschlauches nicht mit der entkalkten, an der Oberfläche ausstreichenden Grundmoräne von Gosda (ANDERs et al. 1989) und mit der an der Ostböschung des Südrandschlauches mehrere Dekameter mächtigen, das Tranitz-Fluviatil abschneidenden und tief in die Gosda-Klinger Rinne eingesenkten Grundmoräne mit drenthezeitlichen Kleingeschiebespektren und vergleichsweise häufigen ViviparusFunden zu konnektieren. Die hier besonders gut erhaltenen und erstaunlich großen Viviparus-Exemplare stecken im Geschiebemergel und treten auch in den an der Nordflanke der Rinne aufgeschlossenen Vorschüttsanden auf.

Was die stratigraphische Zuordnung der unter dem Tranitz-Fluviatil aufgeschlossenen mächtigen Geschiebemergel-Beckensediment-Abfolge anbelangt, so sehen wir in den Ergebnissen der von Hellwig und der ersten durch uns durchgeführten Geschiebeanalysen (u. a. hohe Ffr- und NK-Werte, $\mathrm{PKgr} / \mathrm{Pkro}+$ sw-Werte $<10$ ), ungeachtet weiterer noch ausstehender Bearbeitungen und trotz der ausgespülten Viviparus-Exemplare, zur Zeit kein überzeugendes Argument für eine Einstufung ins SI-Glazial sensu CePEK bzw. ins Drenthe-Stadium. Vielmehr sprechen die durch Mehrfachvorbelastung überwiegend "betonartige" Konsistenz der Grundmoräne („Betonmoräne“), ihre glazitektonisch bedingte brekziöse Struktur und auch die Ergebnisse der Kleingeschiebeanalysen, auch der HELLWIGschen, zumindest unterhalb der Beckensedimente, für eine Zuordnung zur Elster-Kaltzeit. Diese Grundmoräne lässt sich nach Süden - allerdings in deutlich reduzierter Mächtigkeit, weil außerhalb der Bohrauer Rinne gelegen und stets hochliegendem Miozän auflagernd - über die Ostrandprofile bei Gosda bis hin in den Südrandschlauch bei Klinge verfolgen und ist dort mit der 
entkalkten und abschnittsweise stark mit Tertiärmaterial durchsetzten Grundmoräne im Liegenden des Tranitz-Fluviatils zu konnektieren. Äquivalente der massiven und überwiegend "betonharten " Grundmoränenausbildung, wie sie hier im Ostrandschlauch bei Mulknitz entwickelt ist, waren noch bis 1998 auch im ehemaligen Tagebau Greifenhain (4350 der TK 25) als so genannte „Betonmoräne“ aufgeschlossen und wurde als „Liegendmoräne" auch im Vorfeld des Tagebaus WelzowSüd mehrfach erbohrt (4451 der TK 25). Die dort durchgeführten geschiebeanalytische Untersuchungen erbrachten sowohl für Greifenhain wie auch für Welzow Kleingeschiebespektren, die den Spektren von Mulknitz sehr ähnlich sind und für die dort ebenfalls ein elsterzeitliches Alter wahrscheinlich gemacht wurde (Lippstreu et al. 1995), das inzwischen auch allgemein akzeptiert wird.

Der aus unseren Dokumentationen und Untersuchungen zu folgernde Hiatus zwischen der genetisch mit der Auffüllung der Dubrau-Bohrauer Rinne verknüpften lagerungsgestörten Sedimentabfolge („Obere Rinnenfolge") und dem TranitzFluviatil wird u. a. auch durch die Ausbildung eines durchgehenden, 1 bis $5 \mathrm{~m}$ mächtigen Entkalkungshorizontes ab Oberkante der Oberen Rinnenfolge gestützt. Hinzu kommt ein weitestgehend mit diesem Entkalkungshorizont einhergehender reduktionsbedingter Farbumschlag der originär zumeist dunkelgrauen bis dunkelgraubraunen bindigen Liegendsedimente in olivgrüne bis grünlichblaue Farben. Dieses Phänomen deutlicher Entkalkung, verbunden mit einer reduktionsbedingten Verfärbung (altangelegter Gr-Horizont), ist entlang des gesamten östlichen Jänschwalder Tagebaufeldes vom Südrand des Baruther Tales über Weissagk und Gosda bis hin zum Klinger Becken zu beobachten und tritt fast niveaugleich auch in den Profilen in der Hornoer Hochfläche auf. Sie ist nicht lithologiegebunden, sondern erfasst Geschiebemergel und Schluffe ebenso wie schluffige Sande oder Sande mit umgelagertem Tertiär. Erwähnung verdient in diesem Zusammenhang auch das 1988 im Bereich der Weißagker Hochfläche beobachtete Auftreten organischer Schmitzen (humifizierte Wurzelreste?) in der vom Bagger frisch angeschnittenen grünlichblauen „Verwitterungs"-rinde auf Grundmoräne. Es könnte ein Hinweis auf die Ausbildung eines durch fluviatile Erosion gekappten Wurzelbodens sein. Die pollenanalytische Untersuchung einer Probe, entnommen aus einer im überlagernden untersten Abschnitt des Tranitz-Fluviatils auftretenden Schluffmudde-Lage, erbrachte jedoch trotz guter Pollenerhaltung wiederum eine nur subarktische bis arktische Vegetationsentwicklung (ERD 1989, unveröff.). Leider wurde die Problematik seinerzeit nicht weiter verfolgt.

In der Ausbildung des markanten, weitflächig verbreiteten Entkalkungs-(„Verwitterungs“)-Horizontes sehen wir die meteorische Wirkung einer Warmzeit. Dafür in Frage käme nach derzeitigem Kenntnisstand das Holstein-Interglazial oder ein Interglazial des Unteren Saale-Komplexes. Es wäre ein weiterer Beweis dafür, dass die Auffüllung der Bohrauer Rinne einschließlich ihrer Deformation in die Elster-Kaltzeit zu stellen wären und die Basis der saalezeitlichen Abfolge an der Oberkante der Entkalkungszone liegen würde, eine Interpretation, wie wir sie bereits in unseren Publikationen über den Jänschwalder Südrandschlauch in Form eines Geologischen Modells zur Diskussion gestellt hatten (u. a. Lippstreu et al. 1994, Abb. 4). Danach würde die saalezeitliche Sedimentation erst mit der Ablagerung des so genannten „Klinger Fluviatils“, einer insbesondere im Bereich des Jänschwalder Südrandschlauchs verbreiteten periglaziär-fluviatilen bis limnischen Ausbildung des unteren TranitzFluviatils sensu Hellwig beginnen. Die eigentliche Aufschotterung der saalezeitlichen Lausitzer Neiße, d. h. des verbreitet kiesig entwickelten oberen Tranitz-Fluviatils sensu HeLLWIG müsste demzufolge in das ausgehende Saale-Frühglazial (post-Dömnitz) bis einschließlich der Frühphase des 1. saalekaltzeitlichen Eisvorstoßes („Drenthe“) eingeordnet werden und eine ausschließlich kaltklimatisch gesteuerte fluviatile Akkumulation repräsentieren. Gegen unsere Interpretation stünden natürlich die Nowelschen Viviparus-Funde, sollte sich denn ihre Herkunft aus dem „unteren“, mächtigen Geschiebemergel durch weitere, aber nicht ausgespülte Funde verifizieren lassen.

Am Hornoer Berg ist an der aktiven Vorschnittböschung (LAUBAG) derzeit auf einer Länge von 2 Kilometern ein bis zu 25 m mächtiger Sedimentkomplex aufgeschlossen, der nach CEPEK et al. 
(1994) auf der Basis von Bohrungsbearbeitungen die Grundmoränen SII und SIII sensu CEPEK mit zwischengeschalteten Beckenablagerungen repräsentiert. Die Beckensedimente sollen rügenwarmzeitlich entkalkt sein.

Obwohl die saalezeitliche Moränensequenz hier aufgespreizt ist, lässt sie sich nach KÜHNER (DEUQUA-Exkursionsführung 2002) insgesamt doch problemlos mit den bei Mulknitz und im Ostrandschlauch aufgeschlossenen Abfolgen korrelieren. Es treten zwei saalezeitliche Geschiebemergelbänke auf, die vertikal jeweils einen deutlichen faziellen Wechsel erkennen lassen. Obwohl sich ihre Kleingeschiebespektren ähneln, sind sie vorbehaltlich weiterer Untersuchungen dem Drenthe und Warthe der Saale-Kaltzeit zuzuordnen. Zwischengelagert sind dunkelfarbene, abwechselnd tonige und feinsandige, geschichtete, überwiegend kalkfreie Staubeckenschluffe und sich abschnittsweise mit ihnen verzahnende kalkfreie Sande. Die pollenanalytische Untersuchung der Schluffe lässt ihre Interpretation als unter kaltzeitlichen Bedingungen umgelagertes jungtertiäres Sediment zu (STRAHL 2002). Es ergab sich kein Hinweis auf eine Erwärmungsphase zwischen den beiden Moränenbildungen.

Unter dem unteren saalezeitlichen Geschiebemergel lagern hellgraue bis weißgraue, kalkfreie Fein- bis Mittelsande glazifluviatiler bis fluviatiler Genese, die KüHNER (frdl. mdl. Mitt.) als zeitliches Äquivalent der unteren Folge des Tranitz-Fluviatils, vergleichbar dem „Klinger Fluviatil“, interpretiert. Unterlagert wird diese klastische Sequenz auch am Hornoer Berg von elsterkaltzeitlichem Geschiebemergel, der ähnlich den von der südlichen Hochfläche beschriebenen Profilen auch hier starke Verwitterungsanzeichen in Form von Entkalkung aufweist und von seiner Oberfläche her grünlich verfärbt ist.

Eine ausführliche Darstellung zum quartären Schichtenaufbau der Hornoer Hochfläche und seiner Korrelierung mit dem Umfeld, insbesondere zum inzwischen erreichten neuen Kenntnisstand, ist für das Heft 1/2003 der „Brandenburgischen Geowissenschaftlichen Beiträge" (Beitrag R. KüHNER, Vattenfall) vorgesehen.
Weitergehende Schlussfolgerungen für die regionalgeologische Gliederung und die Stratifizierung des Saaleglazials sind im DEUQUA-Exkursionsführer (STACKebrandt et al. 2002) und vor allem in den Erläuterungen zur Geologischen Übersichtskarte des Landes Brandenburg 1:300 000 (Lippstreu et al. 1997) sowie in der stratigraphischen Gliederung des Quartärs (LGRB, LIPPSTREU, zuletzt 2002) niedergelegt.

\section{Schriftenverzeichnis}

Anders, T., Kưhl, A., Lippstreu, L. \& Lohde, H. (1989): Die Gliederung der quartären Schichtenfolgen in den Ostrandböschungen der Tagebaue Cottbus-Nord und Jänschwalde. - Unveröff. Material ZGI; Berlin.

Behrendt, L. (1998): Vergleich der Grundmoränen des Saale-Glazials der Niederlausitz mit denen Nordwestdeutschlands, der Niederlande und Polens. Brandenburgische Geowiss. Beitr., 5 (2): 29-41; Kleinmachnow.

Ceper, A. G., HellwiG, D. \& W. Nowel (1994): Zur Gliederung des Saale-Komplexes im Niederlausitzer Braunkohlenrevier. - Brandenburgische Geowiss. Beitr., 1 (1): 43-83; Kleinmachnow.

ERD, K. (1989): Ergebnisbericht Biostratigraphie Braunkohlenvorfelderkundung. - Unveröff. Ber. ZGI; Berlin.

HellwiG, D. (1975): Fluviatile Bildungen innerhalb des Saale-Komplexes im Raum Cottbus-Forst. - Z. geol. Wiss., 3: 1077-1090; Berlin.

Köhler, E. \& ERD, K. (1980): Pollenanalytische Untersuchung der Bohrungen Brk Müllrose 3/73 und 7/73. - Unveröff. Ber. ZGI; Berlin.

KRBETSCHEK, M. R. \& StOlz, W. (1994): LumineszenzDatierungen an pleistozänen Sedimenten aus Tagebauen des Mitteldeutschen und Lausitzer Braunkohlenreviers. - In: EIsSMANN, L. \&. LrTT, T. [Hrsg.]: Das Quartär Mitteldeutschlands, Exkursionsf. 27. DEUQUA-Tagung in Leipzig. - Altenburger. naturwiss. Forsch., 7: 289-295; Altenburg.

Lippstreu, L. (1998): Kleingeschiebeanalysen von Geschiebemergeln aus dem Vorfeld des Tagebaus Welzow-Süd (LAUBAG). - Unveröff. Ber. LGRB: 3 S., 14 Anl.; Kleinmachnow.

- (2002): Die Gliederung des Pleistozäns im Land Brandenburg. - In: Stackebrandt, W. \& ManHENKE, V. [Hrsg.]: Atlas zur Geologie von Brandenburg: 120-121; Kleinmachnow.

- Hermsdorf, N. \& Sonntag, A. (1995): Zur Gliederung der quartären Sedimentabfolgen im Niederlausitzer Braunkohletagebau Greifenhain (LAUBAG) und in seinem Umfeld. - Brandenburgische Geowiss. Beitr., 2 (1): 61-67; Kleinmachnow. 
- Hermsdorf, N. \& Sonntag, A. (1997): Geologische Übersichtskarte des Landes Brandenburg 1:300 000. - Potsdam, Kleinmachnow.

- Hermsdorf, N., Sonntag, A. \& Thieke, H.U. (1994): Zur Gliederung der quartären Sedimentabfolgen im Niederlausitzer Braunkohlentagebau Jänschwalde und in seinem Umfeld - ein Beitrag zur Gliederung der Saale-Kaltzeit in Brandenburg. Brandenburgische Geowiss. Beitr., 1 (1): 15-35; Kleinmachnow.

LUCKerT, J. \& ThIEKe, H.U. (2000): Der Mineralbestand brandenburgischer Grundmoränen als lithostratigraphischer Indikator - erste Ergebnisse der Röntgenphasenanalyse der Tillmatrix $<63 \mathrm{~m}$. - Brandenburgische Geowiss. Beitr., 7 (1/2): 101-113; Kleinmachnow.

Nowel, W. (1996): Aktuelle Bemerkungen zur Gliederung des Saale-Komplexes im Quartär der Niederlausitz. - Natur und Landschaft der Niederlausitz, 17: 54-77; Cottbus.

- (2003): Zur Korrelation der Glazialfolgen im SaaleKomplex Nord- und Mitteldeutschlands am Beispiel des Tagesbaus Jänschwalde in Brandenburg. - Eiszeitalter und Gegenwart, 52: 47-83; Hannover.
SeIfert, M. (1989): Mitteilung über die pollenanalytische Untersuchung der Bohrung 3919 A3/89 Calau/Süd. - Unveröff. Ber. GFE: 2 S., 1 Abb., 2 Tab.; Freiberg.

Stackebrandt, W., Beer, H., Heinrich, W.-D., HermsDORf, N., Jubitz, K.-B., Koszinski, A., Kühner, R., Lippstreu, L., Streichan, H.-J., Striegler, R. \& STRIEGLer, U. (2002): Exkursion A2, Großabbaue in Brandenburg. - In: ReißMAnN, C. \&. BÖSE, M. [Hrsg.]: Exkursionsführer DEUQUA 2002: 37-52; Berlin/Potsdam.

STRAHL, J. (2002): Bericht über die pollenanalytische Untersuchung von 2 Proben aus dem Tagebau Jänschwalde, Vorschnittböschung Hornoer Berg, Land Brandenburg. - Unveröff. Ber. LGRB: 3 S., 1 Anl.; Kleinmachnow.

TGL 25232 (1980): Analyse des Geschiebebestandes quartärer Grundmoränen. - Fachbereichsstandard, Bl. 01-05, Staatssekr. f. Geologie der DDR, Berlin.

Unger, K. P., Hübner, F. \& Escher, D. (1995): Holstein- und Eem-Interglaziale im Bereich des Lausitzer Grenzwalls und die Gliederung der Saalevereisung zwischen Baruther und Lausitzer Urstromtal. Eiszeitalter und Gegenwart, 45: 24-31; Hannover. 